\title{
AUTOMORPHISMS OF GROUP EXTENSIONS
}

\author{
BY \\ CHARLES WELLS
}

\begin{abstract}
If $1 \rightarrow G \stackrel{\iota}{\rightarrow} E \stackrel{n}{\rightarrow} \Pi \rightarrow 1$ is a group extension, with ı an inclusion, any automorphism $\varphi$ of $E$ which takes $G$ onto itself induces automorphisms $\tau$ on $G$ and $\sigma$ on $\Pi$. However, for a pair $(\sigma, \tau)$ of automorphism of $\Pi$ and $G$, there may not be an automorphism of $E$ inducing the pair.

Let $\bar{\alpha}: \Pi \rightarrow$ Out $G$ be the homomorphism induced by the given extension. A pair $(\sigma, \tau) \in$ Aut $\Pi \times$ Aut $G$ is called compatible if $\sigma$ fixes ker $\bar{\alpha}$, and the automorphism induced by $\sigma$ on $\Pi \bar{\alpha}$ is the same as that induced by the inner automorphism of Out $G$ determined by $\tau$. Let $C<$ Aut $\Pi \times$ Aut $G$ be the group of compatible pairs. Let Aut $(E ; G)$ denote the group of automorphisms of $E$ fixing $G$. The main result of this paper is the construction of an exact sequence
\end{abstract}

$$
1 \rightarrow Z_{\alpha}^{1}(\Pi, Z G) \rightarrow \text { Aut }(E ; G) \rightarrow C \rightarrow H_{\alpha}^{2}(\Pi, Z G) .
$$

The last map is not surjective in general. It is not even a group homomorphism, but the sequence is nevertheless "exact" at $C$ in the obvious sense.

1. Notation. If $G$ is a group with subgroup $H$, we write $H<G$; if $H$ is normal in $G, H \triangleleft G . C_{G} H$ and $N_{G} H$ are the centralizer and normalizer of $H$ in $G$. Aut $G$, Inn $G$, Out $G$, and $Z G$ are the automorphism group, the inner automorphism group, the outer automorphism group, and the center of $G$, respectively. If $\tau \in$ Aut $G, \bar{\tau}$ denotes its natural image in Out $G$. If $g \in G$, Ig denotes the inner automorphism of $G$ induced by $g$ : for $h \in G, h I g=h^{g}=g^{-1} h g$.

Maps will be written on the right; composition reads from left to right. If $S$ and $T$ are sets, $S^{T}$ denotes the set of maps from $T$ to $S$. It is sometimes convenient to write functions exponentially; e.g., $x^{\varphi}$ for $x \varphi$.

Throughout the paper, we shall consider fixed groups $G, E, \Pi$ for which $0 \rightarrow G \stackrel{\iota}{\rightarrow} E \stackrel{n}{\rightarrow} \Pi \rightarrow 0$ is exact; $G$ and $E$ will be written in additive notation, $\Pi$ multiplicatively. $G$ will be identified with its image $G \iota<E$. We fix a left transversal $T: \Pi \rightarrow E$, specifying $1 T=0$. Changing the transversal, and omitting the requirement $1 T=0$ has an effect on almost every statement herein, but the effect can always be calculated explicitly and is ignored. (See Hall [9], Mac Lane [14], or Rédei [17].) $T$ can be chosen so that it is a homomorphism if and only if $E$ splits over $G$.

Every element of $E$ can be written uniquely as $x T+a$ for some $x \in \Pi, a \in G$. (When one writes functions on the left, the form $a^{\prime}+x T$ is better.) For $x \in \Pi$,

Presented to the Society, January 21, 1971; received by the editors April 21, 1970.

AMS 1969 subject classifications. Primary 2022, 2048; Secondary 2050.

Key words and phrases. Automorphism, extension, Schreier factor function, cohomology group. 
$a \in G$, we write $a x$ for $-x T+a+x T$. We have a map $\alpha: \Pi \rightarrow$ Aut $G$ defined by $a x=a^{x \alpha}$. We set $\bar{\alpha}: \Pi \rightarrow$ Out $G$ to be the composition of $\alpha$ with the natural map Aut $G \rightarrow$ Out $G$. Unlike $\alpha, \bar{\alpha}$ is independent of $T$ and is always a homomorphism (see (1.3) below).

For $a, b \in G, x, y \in \Pi$, we have

$$
x T+a+y T+b=(x y) T+\mu(x, y)+a y+b
$$

for the Schreier factor function $\mu: \Pi^{2} \rightarrow G$. This function satisfies the following relations:

$$
\begin{array}{cl}
\mu(x, y z)+\mu(y, z)=\mu(x y, z)+\mu(x, y) z & (x, y, z \in \Pi), \\
(a x) y=[a(x y)]^{\mu(x, y)}=-\mu(x, y)+a(x y)+\mu(x, y) & (a \in G, x, y \in \Pi) .
\end{array}
$$

2. Statement of the Theorem. Let $\varphi \in \operatorname{Aut}(E ; G)$. Then $\varphi$ induces automorphisms $\tau \in$ Aut $G$ and $\sigma \in$ Aut $\Pi$. The induction map Aut $(E ; G) \rightarrow$ Aut $\Pi \times$ Aut $G$ is a group homomorphism. The induction map is not in general surjective; a pair $(\sigma, \tau)$ which is induced by an automorphism $\varphi \in$ Aut $(E ; G)$ is called inducible.

As is customary, $Z_{\alpha}^{1}(\Pi, Z G)$ will denote the group of mappings (crossed homomorphisms or 1-cocycles) $\gamma: \Pi \rightarrow Z G$ satisfying

$$
(x y) \gamma=x \gamma y+y \gamma \quad(x, y \in \Pi) .
$$

(It follows that $1 \gamma=0$.) Each $\gamma \in Z_{\alpha}^{1}(\Pi, Z G)$ induces an automorphism $\varphi$ fixing $G$ and $\Pi$ elementwise, defined by the formula

$$
(x T+a) \varphi=x T+x \gamma+a \quad(x \in \Pi, a \in G) .
$$

This gives a map $Z_{\alpha}^{1}(\Pi, Z G) \rightarrow$ Aut $(E ; G)$.

Also, let $C$ be the subgroup of Aut $\Pi \times$ Aut $G$ of compatible automorphisms as defined in the abstract, and let $H_{\alpha}^{2}(\Pi, Z G) \cong \operatorname{Ext}(\Pi, G)$ be the group of extension classes of $G$ by $\Pi$ with the given homomorphism $\bar{\alpha}$ (Mac Lane [14, p. 128]).

We may now state the main result.

THEOREM. There is a set map $C \rightarrow H_{\alpha}^{2}(\Pi, Z G)$ such that the sequence

$$
1 \rightarrow Z_{\alpha}^{1}(\Pi, Z G) \rightarrow \text { Aut }(E ; G) \rightarrow C \rightarrow H_{\alpha}^{2}(\Pi, Z G)
$$

is exact. The middle two maps are the induction maps defined in the preceding paragraphs.

Note 1. This Theorem is related to the well-known theorem that Ext repairs the inexactness of Hom on the right. We have associated to each $(\sigma, \tau) \in C$ an element of $H_{\alpha}^{2}(\Pi, Z G)$ so that $(\sigma, \tau)$ is associated to 0 if and only if it is inducible. But one can think of $\tau$ as being in $\operatorname{Hom}(G, E)$. If $(\sigma, \tau)$ is inducible, then $\tau$ can be extended to $\operatorname{Hom}(E, E)$.

However, here we want to extend $\tau$ not merely to $\operatorname{Hom}(E, E)$, but to Aut $E$, and in such a way that $\sigma$ is induced on $\Pi$. Moreover, the group structure on $C$ is 
composition, not pointwise addition. So it is not surprising that the map $C \rightarrow H_{\alpha}^{2}(\Pi, Z G)$ is not a homomorphism.

Note 2. A useful example is a cyclic group $C_{m n}$ regarded as an $\left\llcorner\right.$ xtension of $C_{m}$ by $C_{n}$. If $d$ is the greatest common divisor of $m$ and $n$, a pair of automorphisms $g \mapsto g^{r}$ of $C_{m}$ and $h \mapsto h^{s}$ of $C_{n}$ is inducible if and only if $d$ divides $r-s$. On the other hand, $C=$ Aut $\Pi \times$ Aut $G$ in this case. A proper choice of $m$ and $n$ shows that it is possible for $(\sigma, \tau)$ and $\left(\sigma^{\prime}, \tau\right)$ both to be compatible while one is inducible and the other is not. A similar statement is true for a change in $\tau$ while keeping $\sigma$ fixed.

Note 3. The structure theorem for Aut $(E ; G)$ implied by the Theorem is a generalization of a number of special results in the literature. See [1], [2], [5], [7], [10], [11], [16]. In many special cases Aut $(E ; G)$ splits over $Z_{\alpha}^{1}(\Pi, Z G)$; it would be interesting to know precisely under what conditions this happens.

NoTE 4. Baer stated (in other words) that inducible pairs are compatible for the case $G$ Abelian in $[3, \S 2$, Folgerung 1]. He also gives a way of calculating when a compatible pair is obstructed in that case. In $\$ 3$ of [3], he characterizes the compatible pairs as precisely those induced by automorphisms of $H_{\alpha}^{2}(\Pi, G)$ (again $G$ is Abelian).

3. Proof of the Theorem. Let $R_{E} G$ denote the group of right translations of elements of $E$ by elements of $G$. Aut $(E ; G)$ consists exactly of the automorphisms of $E$ that normalize $R_{E} G$. Suppose $\varphi \in$ Aut $(E ; G)$. Now,

$$
x T_{\varphi}=x^{\sigma} T+x \gamma_{1} \quad(x \in \Pi)
$$

for maps $\sigma: \Pi \rightarrow \Pi$ and $\gamma_{1}: \Pi \rightarrow G$. For any $a \in G, x T+a$ is obtained from $x T$ by an element of $R_{E} G$, so there is a map $\tau \in$ Aut $G$ for which

$$
(x T+a) \varphi=x^{\sigma} T+x \gamma_{1}+a^{\tau} \quad(x \in \Pi, a \in G) .
$$

It follows immediately that $\sigma$ is a surjective homomorphism. If $x^{\sigma} T=y^{\sigma} T$, then $x T \varphi=\left(y T-\left(y \gamma_{1}\right)^{z^{-1}}+\left(x \gamma_{1}\right)^{z^{-1}}\right) \varphi$, so $x=y$; hence $\sigma$ is also injective.

Let $\gamma=\sigma^{-1} \gamma_{1}$; then

$$
(x T+a) \varphi=x^{\sigma} T+x^{\sigma} \gamma+a^{\tau} \quad(x \in \Pi, a \in G),
$$

and $\varphi$ determines $\gamma$ uniquely, since $T$ is fixed. (The $\gamma_{1}$ here is the same as $\gamma$ in [19] and [2]; the change in notation makes some formulas simpler.)

Lemma. There is a bijection between Aut $(E ; G)$ and the elements $(\sigma, \tau, \gamma) \in$ Aut $\Pi$ $\times$ Aut $G \times G^{\Pi}$ which satisfy

$$
(x y) \gamma=\mu(x, y)+x \gamma y+y \gamma-\mu\left(x^{\sigma^{-1}}, y^{\sigma-1}\right)^{\tau} \quad(x, y \in \Pi)
$$

and

$$
a^{\tau} y=y \gamma+\left(a y^{\sigma-1}\right)^{\tau}-y \gamma \quad(a \in G, y \in \Pi) .
$$


Under this bijection, $\varphi \in$ Aut $(E ; G)$ is associated with the triple $(\sigma, \tau, \gamma)$ in which $\sigma$ is the automorphism of $\Pi$ induced by $\varphi, \tau=\varphi \mid G$, and

$$
x y=-x T+x^{\sigma^{-1}} T \varphi \quad(x \in \Pi) .
$$

Conversely, $(\sigma, \tau, \gamma) \in$ Aut $\Pi \times$ Aut $G \times G^{\Pi}$ is associated to the map $\varphi$ defined by (3.1).

Proof. Assume $\varphi \in$ Aut $(E ; G)$. Applying $\varphi$ respectively to the sums $x^{\sigma^{-1}} T+y^{\sigma-1} T$ ( $x, y \in \Pi)$ and $a+y^{\sigma-1} T(a \in G, y \in \Pi)$ and using (1.1) and (3.1) yields (3.2) and (3.3). Conversely, one may calculate directly that if $(\sigma, \tau, \gamma)$ satisfies (3.2) and (3.3) then the map $\varphi$ defined by $(3.1)$ is in Aut $(G ; E)$.

We now proceed to prove the Theorem. First, note that (3.3) immediately implies that the induction map Aut $(E ; G) \rightarrow$ Aut $\Pi \times$ Aut $G$ actually has image in $C$. (Every inducible pair is compatible.)

We next show that the sequence of the Theorem is exact at Aut $(E ; G)$. In the first place, the second map of the sequence takes $\gamma \in Z_{\alpha}^{1}(\Pi, Z G)$ onto the automorphism in Aut $(E ; G)$ corresponding to $(1,1, \gamma)$, so the composite across Aut $(E ; G)$ is 0 . Secondly, assume $(1,1, \gamma)$ corresponds to an automorphism of $E$ that fixes $G$. Then (3.3) for the case $\sigma=\tau=1$ yields the fact that $\Pi \gamma<Z G$. Using this, (3.2) immediately implies that $\gamma \in Z_{\alpha}^{1}(\Pi, Z G)$.

We now construct a map $C \rightarrow G^{\Pi \times \Pi}$ which will induce the right-most map of the Theorem.

Let $(\sigma, \tau) \in C$; then by definition, for any $y \in \Pi$, there is an inner automorphism $j$ of $G$ depending on $y$ such that, for all $a \in G$,

$$
a\left(y^{\sigma}\right)=\left(a^{\tau-1} y\right)^{\tau j} .
$$

Let $\gamma: \Pi \rightarrow G$ be a map with the property that $j$ is the inner automorphism induced by $-y^{\sigma} \gamma$. It follows that $(\sigma, \tau, \gamma)$ satisfies (3.3). We then associate to $(\sigma, \tau)$ the map $k: \Pi \times \Pi \rightarrow G$ which measures how $\gamma$ deviates from satisfying (3.2). Specifically,

$$
k(x, y)=(x y) \gamma+\mu\left(x^{\sigma^{-1}}, y^{\sigma-1}\right)^{\tau}-y \gamma-x \gamma y-\mu(x, y) \quad(x, y \in \Pi) .
$$

The following four steps then complete the proof of the Theorem.

1. Im $k \subset Z G$. It follows from (3.3) that for any $a \in G,\left(a\left(x^{\sigma^{-1}} y^{\sigma^{-1}}\right)\right)^{\tau}$ is obtained from $a^{\tau}(x y)$ by conjugation by $(x y) \gamma$. However, it also follows by applying first (1.3) and then (3.3) that one gets the same result by conjugation by $\mu(x, y)+x \gamma y$ $+y_{\gamma}-\mu\left(x^{\sigma^{-1}}, y^{\sigma^{-1}}\right)^{\tau}$. Therefore, $k(x, y)$, the difference of these two elements, is in $Z G$.

2. The function $k$ is a 2-cocycle. First,

$$
\begin{aligned}
-k(x y, z)+k(x, y z)= & \mu(x y, z)+(x y) \gamma z+z \gamma-\mu\left(x y^{\sigma-1}, z^{\sigma^{-1}}\right)^{\tau} \\
& +\mu\left(x^{\sigma-1},(y z)^{\sigma-1}\right)^{\tau}-(y z) \gamma-x \gamma(y z)-\mu(x, y z)
\end{aligned}
$$

$(x, y, z \in \Pi)$. 
Apply (1.2) to the fourth and fifth terms, and (1.3) to the penultimate term. Then apply (3.3) to the (new) fourth term and (1.2) to the last two terms. The right side of (3.7) is now

$$
\begin{aligned}
\mu(x y, z)+ & (x y) \gamma z+\mu\left(x^{\sigma^{-1}}, y^{\sigma^{-1}}\right)^{\tau} z+z \gamma-\mu\left(y^{\sigma-1}, z^{\sigma^{-1}}\right)^{\tau} \\
& -(y z) \gamma+\mu(y, z)-(x \gamma y) z-\mu(x, y) z-\mu(x y, z) \quad(x, y, z \in \Pi) .
\end{aligned}
$$

Use (3.6) to replace the second and third terms of (3.8) by $k(x, y) z+\mu(x, y) z$ $+(x \gamma y) z+y \gamma z$, and the fourth, fifth and sixth terms by $-y \gamma z-\mu(y, z)-k(y, z)$. It then follows from the fact that $\operatorname{Im} k \subset Z G$ that (3.8) is $k(x, y) z-k(y, z)$. Thus $k$ is a 2-cocycle.

3. Changing $\gamma$ changes $k$ by a coboundary. Suppose $\left(\sigma, \tau, \gamma^{\prime}\right)$ also satisfies (3.3). Let $k^{\prime}(x, y)$ be defined by (3.6) with $\gamma^{\prime}$ in place of $\gamma$. We shall show that $k(x, y)$ $-k^{\prime}(x, y)$ is the coboundary of $\gamma-\gamma^{\prime}$ (whose image is clearly within $Z G$ ). By using (3.6) and the fact that, for $a, b \in G, a+b=b^{-a}+a$, we have

$$
k(x, y)-k^{\prime}(x, y)=(x y) \gamma-\left[(x y) \gamma^{\prime}\right]^{g}-y \gamma+\left(y \gamma^{\prime}\right)^{h}-x \gamma y+x \gamma^{\prime} y \quad(x, y \in \Pi)
$$

for certain elements $g, h \in G$ which an easy calculation shows are in $\operatorname{Im}\left(\gamma-\gamma^{\prime}\right)$ $\subset Z G$. This proves that $k-k^{\prime}$ is a coboundary.

4. Any cocycle cohomologous to $k$ arises from some $\gamma$. Let $k^{\prime \prime}=k+\delta f$, i.e. $k^{\prime \prime}(x, y)=k(x, y)+(x y) f-x f y-y f$, for some $f: \Pi \rightarrow Z G$. If $\gamma+f$ is put in the right side of (3.6) in place of $\gamma$, then the left side becomes $k^{\prime \prime}$. But $\gamma+f$ satisfies (3.3) when $\gamma$ does, so this proves the assertion.

Since it is clear that $k$ can be chosen to be 0 if and only if $(\sigma, \tau)$ is inducible, we have now proved that the map that takes $(\sigma, \tau)$ onto the cohomology class of $k$ is well defined and that its "kernel" is exactly the collection of inducible pairs of automorphisms in $C$. This proves the Theorem.

The author wishes to acknowledge the useful comments and suggestions of J. Altinger, J. Duskin, D. H. Lee, and the referee.

\section{REFERENCES}

1. J. E. Adney and W. E. Deskins, On automorphisms and subgroups of finite groups. II, Arch. Math. (Basel) 18 (1967), 1-7. MR 34 \#5951.

2. J. Altinger, Normalizers of permutation groups, Dissertation, Case Western Reserve University, Cleveland, Ohio, 1969.

3. R. Baer, Erweiterung von Gruppen und ihren Isomorphismen, Math. Z. 38 (1934), 375-416.

4. - Automorphismen von Erweiterungsgruppen, Hermann, Paris, 1935.

5. O. Chein, IA automorphisms of free and free metabelian groups, Comm. Pure Appl. Math. 21 (1968), 605-629. MR 39 \#1537.

6. J. Dauns and K. H. Hofmann, Nilpotent groups and automorphisms, Acta Sci. Math. (Szeged) 29 (1968), 225-246. MR 38 \#2200.

7. J. A. Green, On the number of automorphisms of a finite group, Proc. Roy. Soc. London Ser. A 237 (1956), 574-581. MR 18, 464.

8. F. Haimo, Normal automorphisms and their fixed points, Trans. Amer. Math. Soc. 78 (1955), 150-167. MR 16, 794.

9. M. Hall, Jr., The theory of groups, Macmillan, New York, 1959. MR 21 \#1996. 
10. J. G. Harvey, Complete holomorphs and chains in partially ordered groups, Dissertation, Tulane University, New Orleans, La., 1961; University Microfilms \#61-3778, Ann Arbor, Michigan.

11. J. C. Howarth, Automorphisms of a finite Abelian group which reduce to the identity on a subgroup or factor group, Proc. Amer. Math. Soc. 12 (1961), 422-427. MR 23 \#A3169.

12. B. Huppert, Endliche Gruppen. I, 2nd ed., Die Grundlehren der math. Wissenschaften, Band 134, Springer-Verlag, Berlin and New York, 1967. MR 37 \#302.

13. A. G. Kuros, Theory of groups, GITTL, Moscow, 1953; English transl., Chelsea, New York, 1960. MR 15, 501; MR 22 \#727.

14. S. Mac Lane, Homology, Die Grundlehren der math. Wissenschaften, Band 114, Academic Press, New York; Springer-Verlag, Berlin, 1963. MR 28 \#122.

15. A. Mader, A note on direct and semi-direct products of groups, Math. Z. 95 (1967), 272-275. MR 34 \#5908.

16. W. H. Mills, The automorphisms of the holomorph of a finite abelian group, Trans. Amer. Math. Soc. 85 (1957), 1-34. MR 19, 387.

17. L. Rédei, Algebra. Vol. I, Akad. Kiadó, Budapest, 1954; English transl., Pergamon Press, New York, 1967. MR 16, 559; MR 35 \#2697.

18. W. R. Scott, Group theory, Prentice-Hall, Englewood Cliffs, N. J., 1964. MR 29 \#4785.

19. C. Wells, H-split translations of groups, J. Algebra 12 (1969), 195-206. MR 39 \#296.

Case Western Reserve University,

Cleveland, Оhio 44106 